\title{
miR-223 enhances the sensitivity of non-small cell lung cancer cells to erlotinib by targeting the insulin-like growth factor-1 receptor
}

\author{
FENG-YI ZHAO $^{1}$, JING HAN ${ }^{1}$, XIE-WAN CHEN ${ }^{2}$, JIANG WANG $^{1}$, XU-DONG WANG ${ }^{1}$, \\ JIAN-GUO SUN ${ }^{1}$ and ZHENG-TANG CHEN ${ }^{1}$ \\ ${ }^{1}$ Cancer Institute of PLA, Xinqiao Hospital, Third Military Medical University, Chongqing 400037; ${ }^{2}$ Medical English Department, \\ College of Basic Medicine, Third Military Medical University, Chongqing 400038, P.R. China
}

Received October 11, 2015; Accepted April 28, 2016

DOI: $10.3892 / \mathrm{ijmm} .2016 .2588$

\begin{abstract}
Lung cancer is the leading cause of cancer-related fatalities worldwide, and non-small cell lung cancer (NSCLC) is the main pathological type. MicroRNAs (miRNAs or miRs) are a class of small non-coding RNAs, which are involved in tumor initiation and progression. miR-223 is a tumor suppressor miRNA that has been reported in various types of cancer, including lung cancer. In the present study, to characterize the biological behavior of miR-223 in NSCLC, we established an miR-223 overexpression model in erlotinibresistant PC-9 (PC-9/ER) cells by infection with lentivirus to induce the overexpression of miR-223. As a result, miR-223 enhanced the sensitivity of the PC-9/ER cells to erlotinib by inducing apoptosis in vitro. Additionally, in vivo experiments were performed using nude mice which were injected with the cancer cells [either the PC-9 (not resistant), PC-9/ER, or the PC-9/ER cells infected with miR-223)]. We found that the tumor volumes were reduced in the rats injected with the cells infected with miR-223. To further explore the underlying mechanisms, reverse transcription-quantitative polymerase chain reaction (RT-qPCR) and western blot analysis were used to identify the target molecules of miR-223. miR-223 was demonstrated to act as a local regulator of insulin-like growth factor-1 receptor (IGF-1R) in the acquired resistance to tyrosine kinase inhibitors (TKIs). Notably, the overexpression of IGF-1R in NSCLC was downregulated by miR-223, and the activation of Akt/S6, the downstream pathway, was also inhibited. The inhibition of IGF-1R by miR-223 was attenuated by exogenous IGF-1 expression. Therefore, miR-223 may regulate the
\end{abstract}

Correspondence to: Professor Zheng-Tang Chen or Professor Jian-Guo Sun, Cancer Institute of PLA, Xinqiao Hospital, Third Military Medical University, 183 Xinqiao Center Street, Shapingba, Chongqing 400037, P.R. China

E-mail:czt05@163.com

E-mail: sunjg09@aliyun.com

Key words: non-small cell lung cancer, insulin-like growth factor-1 receptor, miR-223, erlotinib, targeted molecular therapy acquired resistance of PC-9/ER cells to erlotinib by targeting the IGF-1R/Akt/S6 signaling pathway. The overexpression of miR-223 may partially reverse the acquired resistance to epidermal growth factor receptor-TKIs, thus, providing a potential therapeutic strategy for TKI-resistant NSCLC.

\section{Introduction}

Lung cancer remains a leading cause of cancer-related mortality worldwide. Non-small cell lung cancer (NSCLC) accounts for $80-85 \%$ of all cancer cases, with an overall 5-year survival rate of $<20 \%(1,2)$. Targeted molecular therapy and small-molecule competitive epidermal growth factor receptor (EGFR) tyrosine kinase inhibitors (TKIs), which largely improve the prognosis of patients, has optimized the therapeutic strategy. Approximately $25 \%$ of NSCLC patients harboring the EGFR active mutation (including exon 19 del and exon 21 L858R) benefit from TKIs (3). However, acquired resistance to TKIs inevitably occurs following 8-10 months of treatment. The acquired resistance positively correlates with a secondary T790M mutation or amplification of hepatocyte growth factor receptor (HGFR) $(3,4)$. In addition, previous studies have demonstrated that the overexpression of insulin-like growth factor-1 receptor (IGF-1R) is also involved in this type of drug resistance (5). Recently, a class of small non-coding RNAs, termed microRNAs (miRNAs or miRs), has also been shown to play important roles in NSCLC (6). However, whether miRNAs are related to resistance to EGFR-TKIs remains unknown. The exact mechanisms involved, thus need to be explored. At the same time, solutions to improve the sensitivity to TKIs are urgently required.

miRNAs are single-stranded RNAs, 20-22 nucleotides in length (7). They usually act as negative regulators of gene expression at the post-transcriptional level (8), and are involved in several cellular functions, including differentiation, proliferation and apoptosis (9-12). Garofalo et al (13) demonstrated that numerous miRNAs play important roles in modulating the cell phenotype of NSCLC. miR-223 is a tumor suppressor miRNA that has been reported in various types of cancer. It has been shown to be expressed at low levels in hepatocellular carcinoma and ovarian cancer $(14,15)$. In our previous study, a low expression level of miR-223 was observed in Lewis lung carcinoma (LLC) tissue, and miR-223 was shown to be 
extensively involved in the cell cycle regulation. Furthermore, the upregulation of miR-223 inhibited the migration and invasion of LLC cells in vitro by targeting IGF-1R (16). miR-223 is involved in regulating a variety of malignant phenotypes. However, the mechanisms through which miR-223 regulates the resistance of NSCLC cells to erlotinib remain unknown.

IGF-1R is a member of the insulin receptor (IR) family, which is well known for its role in the resistance of NSCLC cells to EGFR-TKIs (17). IGF-1R is highly expressed in lung adenocarcinoma, breast cancer, prostatic cancer and pancreatic carcinoma (18). The intracellular signaling of IGF-1R is mediated by IGF-1, which in turn results in the activation of the mitogen-activated protein kinase (MAPK) and phosphatidylinositol 3-kinase (PI3K)/Akt pathways (19). PI3K/Akt is considered to be predominant in the resistance to EGFR-TKIs. miR-223 has been shown to suppress cell proliferation by regulating the IGF-1R signaling pathway in HeLa cells (15). Moreover, miR-223 plays a central role in degranulation by targeting the IGF-1R/PI3K/Akt pathway in mast cells (20). Based on the above-mentioned findings, we hypothesized that miR-223 may modulate apoptosis and improve the sensitivity of NSCLC cells to erlotinib by targeting the IGF-1R/Akt signaling pathway. In the present study, miR-223 was overexpressed in erlotinib-resistant PC-9 (PC-9/ER) cells and the potential regulatory effects of miR-223 on IGF-1R were investigated.

\section{Materials and methods}

Cell lines, reagents and groups. The lung adenocarcinoma PC-9 cell line was purchased from the American Type Culture Collection (ATCC, Manassas, VA, USA), and cultured in RPMI1640 medium, with $10 \%$ fetal bovine serum (FBS) (both from Gibco; Thermo Fisher Scientific, Inc., Waltham, MA, USA) and $100 \mathrm{U} / \mathrm{ml}$ penicillin/streptomycin (Sigma-Aldrich, St. Louis, MO, USA). All cells were cultured at $37^{\circ} \mathrm{C}$ in a humidified atmosphere of $95 \%$ air $/ 5 \% \mathrm{CO}_{2}$. Erlotinib (Sigma-Aldrich) was dissolved by dimethyl sulfoxide (DMSO) and stored at $-20^{\circ} \mathrm{C}$. The concentration used to maintain the resistance of PC-9/ER cells to erlotinib was $0.1 \mu \mathrm{M}$. The cells were divided into 6 groups as follows: PC-9, PC-9/ER, PC-9/ER-miR-223, PC-9/ER-miR-223 plus IGF-1, PC-9/ER-empty vector (EV) and PC-9/ER-EV plus IGF-1.

Establishment of erlotinib-resistant PC-9/ER cell line. The PC-9 cell line harboring exon 19 del mutation acquired erlotinib resistance following 6 months of continuous drug exposure $(1 \mu \mathrm{M}$ erlotinib). The cell counting kit-8 (CCK-8; Dojindo, Kumamoto, Japan) was used to confirm the resistance of the PC-9 cells to erlotinib (PC-9/ER), and this was confirmed 3 times. The cells were allowed to grow under drug-free conditions for at least 1 week before being used in the experiments. The PC-9/ER cells did not harbor the T790M mutation, according to our subsequent EGFR gene detection (data not shown).

Infection with lentivirus. The lentiviral vector GV259, used to induce miR-223 overexpression, was packaged and purchased from Shanghai GeneChem Co., Ltd. (Shanghai, China). The PC-9/ER cells $\left(5 \times 10^{4}\right)$ were infected with $2 \times 10^{6}$ lentivirus-transducing units in the presence of $10 \mu \mathrm{g} / \mathrm{ml}$ polybrene in a 24 -well plate $(\mathrm{MOL}=40)$. An empty lentiviral vector was transfected into the target cells as the control under identical conditions. The EV group is the control of miR-223 group both in vivo and in vitro. Therefore, the infected cells were referred to as either miR-223/EV or as PC-9/ER-miR-223/PC-9/ER-EV, respectively. The cells were collected and the media were updated following cultivation for $12 \mathrm{~h}$. The transfection efficiency was observed under a fluorescent microscope (BX50; Olympus, Tokyo, Japan). The transfected cells were subsequently sorted using a fluorescence activated cell sorter (FACS) based on the green fluorescent protein (GFP) signal.

Cell proliferation assay. Cell proliferation assay was carried out according to the instructions of the manufacturer of CCK-8 (Dojindo). Briefly, the PC-9 and PC-9/ER cells were seeded in 96-well plates at a density of $3.5 \times 10^{3}$ cells/well with $100 \mu \mathrm{l}$ cell culture medium. After $24 \mathrm{~h}$ of culture, the medium was removed from the wells and replaced with medium containing erlotinib at concentrations ranging between 0.01 and $500 \mu \mathrm{M}$. Following further culture for $48 \mathrm{~h}, 100 \mu \mathrm{l}$ of medium containing $10 \%$ CCK-8 were added to each well. The cells were then incubated at $37^{\circ} \mathrm{C}$ for approximately $30 \mathrm{~min}$. The absorbance was detected at $450 \mathrm{~nm}$ using a spectrophotometer (Epoch; BioTek, Winooski, VT, USA). All experiments were set up in triplicate, and confirmed in at least 3 independent experiments.

Reverse transcription-quantitative polymerase chain reaction $(R T-q P C R)$. Total RNA was extracted from the cells using RNAiso Plus reagent (Takara Bio, Inc., Otsu, Japan), according to the manufacturer's instructions. The expression of miR-223 was detected by one-step RT-qPCR with SYBR Premix Ex Taq (Takara Bio., Inc.). The specific primers of miR-223 and IGF-1R were designed by Shanghai GeneChem Co., Ltd. The relative expression levels were calculated by means of the $2^{-\Delta \Delta \mathrm{Ct}}$ method, relative to the internal controls, U6 RNA for miRNA and $\beta$-actin for genes, respectively. The following primers were used: IGF-1R forward, 5'-GGCATACCTCAA CGCCAATA-3' andreverse,5'-CAGCCCTTTCCCTCCTTT-3'; $\beta$-actin forward, 5'-GTGAAGGTGACAGCAGTCGGTT-3' and reverse, 5'-GAAGTGGGGTGGCTTTTAGGA-3'. All the RT-qPCR reactions were run in triplicate and repeated in 3 independent experiments.

Western blot analysis. Total protein was extracted from the cells using radio immunoprecipitation assay (RIPA) buffer and was quantified by a bicinchoninic acid assay kit (Beyotime Institute of Biotechnology, Haimen, China). The proteins were separated by electrophoresis on $12 \%$ sodium dodecyl sulfate-polyacrylamide gel electrophoresis (SDS-PAGE) gels and were transferred onto polyvinylidene difluoride membranes (EMD Millipore, Billerica, MA, USA). The membranes were then blocked for $1 \mathrm{~h}$ at room temperature with $5 \%$ BSA and were subsequently incubated overnight at $4{ }^{\circ} \mathrm{C}$ with the following antibodies: Polyclonal rabbit anti-IGF-1R (cat. no. 3027), monoclonal rabbit anti-phosphorylated (p-)IGF-1R (cat. no. 3918), polyclonal rabbit anti-Akt (cat. no. 4691), polyclonal rabbit antip-Akt (cat. no. 4060), monoclonal rabbit anti-S6 (cat. no. 2217), monoclonal rabbit anti-p-S6 (cat. no. 4858) and polyclonal mouse anti- $\beta$-actin (cat. no. 3700; 1:1,000) (Cell Signaling Technology, Inc., Beverly, MA, USA). Following washing 3 times with Tris-buffered saline containing Tween-20 
(1:500), the membranes were incubated with secondary antibodies-anti-rabbit IgG (cat. no. SA00001-2; 1:5,000) and anti-actin antibody (cat. no. 20536-1-AP; 1:5,000) (both from Proteintech Group, Chicago, IL, USA) for $1 \mathrm{~h}$ at room temperature as previously described (21). The blots were visualized using enhanced chemiluminescence, as previously described (22). The density of the bands was quantified using Quantity One software (Bio-Rad Laboratories, Inc., Hercules, CA, USA).

Analysis of apoptosis. A total of $5 \times 10^{5}$ cells was resuspended in phosphate-buffered saline (PBS) following exposure to $1 \mu \mathrm{M}$ erlotinib for $48 \mathrm{~h}$. The cells were stained using the Annexin V-APC/7-AAD Apoptosis Detection kit (KeyGen Biotech, Nanjing, China) and subsequently analyzed using a flow cytometer (Beckman Coulter, Brea, CA, USA) equipped with Summit software according to the manufacturer's instructions.

IGF-1 stimulation experiments in vitro. To induce exogenous IGF-1 expression, the PC-9/ER-miR-223 and PC-9/ER-EV cells were seeded in 6-well plates with RPMI-1640 medium containing $10 \%$ FBS. Once the cells reached $70 \%$ confluence, they were washed twice with serum-free medium to remove the FBS. Following overnight serum starvation, the cells were stimulated for $30 \mathrm{~min}$ at $37^{\circ} \mathrm{C}, 5 \% \mathrm{CO}_{2}$ in serum-free medium containing $100 \mathrm{ng} / \mathrm{ml}$ of IGF-1 (cat. no. CYT-216; ProSpec, Rehovot, Israel), as previously described (17). The cells were washed with $1 \mathrm{X}$ ice-cold PBS and incubated on ice in RIPA lysis buffer or RNAiso Plus reagent to continue the experiment.

In vivo experiments. Nude mice (male, 4-6 weeks old) were purchased from the Chinese Academy of Medical Sciences (Beijing, China). They were housed in a laminar flow room under specific pathogen-free conditions at room temperature $\left(22 \pm 2^{\circ} \mathrm{C}\right)$ and humidity $(<40 \%)$ with free access to food and water. In tumor growth assay, a total of 18 mice was randomly divided into 6 groups. A total of $2 \times 10^{6}$ PC-9, PC-9/ER, PC-9/ER-miR-223 and PC-9/ER-EV cells was subcutaneously injected into the lower quadrant of the rats. When the tumor volumes reached approximately $100-150 \mathrm{~mm}^{3}$, erlotinib $(60 \mathrm{mg} / \mathrm{kg})$ was intragastrically administered from the 14th day to each mouse daily for 14 days. Moreover, the mice in the PC-9/ER-miR-223 and PC-9/ER-EV groups received a peritoneal perfusion of IGF-1 $(50 \mu \mathrm{g} / \mathrm{kg})$ daily for 14 days and were classified as groups PC-9/ER-miR-223 plus IGF-1 and PC-9/ER-EV plus IGF-1. We evaluated tumor sizes every 3 days for 4 weeks using calipers. Tumors were calculated according to the formula $\mathrm{V}=\left(\right.$ length $\left.\mathrm{x} \mathrm{width}^{2}\right) / 2$. Additionally, to observe survival, another 30 nude mice were randomly divided into 6 groups in this study. The grouping, erlotinib administration and IGF-1 injection were the same as mentioned above. These mice were not sacrificed by cervical dislocation until they suffered from limitations in activities and significant weight loss. Efforts were made to reduce the animal suffering and the number of animals used. All the animal care and experimentation were approved by the Institutional Animal Care and Use Committee of the Third Military Medical University (Chongqing, China).

Statistical analysis. Quantitative data are presented as the means \pm standard deviation (SD). The Student's t-test was used to analyze data following testing for normal distribution and homoscedasticity. Statistical analysis was performed and the half inhibitory concentration $\left(\mathrm{IC}_{50}\right)$ of erlotinib was calculated using GraphPad Prism 5 (GraphPad Software, Inc., San Diego, CA, USA). A value of $\mathrm{P}<0.05$ was considered to indicate a statistically significant difference. All the experiments were independently repeated at least 3 times.

\section{Results}

Lentivirus-mediated miR-223 overexpression and IGF-1R expression in the PC-9 cell line. Lentiviral gene transfer is capable of inducing a stable gain- and loss-of-function phenotype of cells. It is a critical tool to explore miRNA function in cell culture and animal models (23). In the present study, lentiviral vectors overexpressing miR-223 (lentivirus-miR-223) and EV were transfected into the PC-9/ER cells. The transfected cells were subsequently sorted using a fluorescence activated cell sorter (FACS) based on the green fluorescent protein (GFP) signal. Prior to sorting, the transfection efficiencies were found to be approximately 35 and 55\%, respectively (Fig. 1A). However, through FACS sorting, the GFP-positive subpopulation was highly purified (purity $>95 \%$ ) (Fig. 1B), similar to our previous study (15). Using RT-qPCR, the levels of miR-223 were detected in the PC-9/ER-miR-223 and PC-9/ER-EV cells. The expression of miR-223 in the miR-223 overexpression group was upregulated approximately 16-fold compared with that in the EV group (Fig. 1C). Using RT-qPCR, the mRNA expression of IGF-1R was also detected in the 4 groups of PC-9 cells (Fig. 1D). The expression of IGF-1R was revealed to be significantly increased in the PC-9/ER cells versus the PC-9 cells (3.14-fold). Furthermore, this upregulation was suppressed by the overexpression of miR-223. The expression of total IGF-1R was also determined in the PC-9 cells (Fig. 1E and F). The increased expression of miR-223 in the PC-9/ER cells led to the downregulation of IGF-1R at both the mRNA and protein level.

Overexpression of miR-223 enhances the sensitivity of PC-9/ER cells to erlotinib by inducing apoptosis. The CCK-8 assay revealed the sensitivity of the PC-9 and PC-9/ER cells (or miR-223 and EV) groups to erlotinib. The PC-9/ER cells were clearly more resistant to erlotinib than the PC-9 cells. The overexpression of miR-223 in the PC-9/ER cells markedly enhanced the sensitivity to erlotinib. The $\mathrm{IC}_{50}$ values of the PC-9, PC-9/ER, PC-9/ER-miR-223 and PC-9/ER-EV cells were: 0.25, 5.16, 1.19 and 5.29, respectively (Fig. 2A). However, the addition of $100 \mathrm{ng} / \mathrm{ml}$ of IGF-1 significantly re-enhanced the resistance of the PC-9/ER-miR-223 cells to erlotinib. The $\mathrm{IC}_{50}$ values of the PC-9/ER-miR-223 and PC-9/ER-miR-223 + IGF-1 cells were 1.04 and 4.29, respectively (Fig. 2B). The differences were statistically significant $(\mathrm{P}<0.05)$. In order to explore the mechanisms underlying this phenomenon, the apoptosis of the cells was assessed. The overexpression of miR-223 was revealed to induce the apoptosis of the PC-9/ER cells treated with $1 \mu \mathrm{M}$ erlotinib for $48 \mathrm{~h}$. Following staining with Annexin V-APC and 7-AAD, the number of apoptotic cells was detected and we observed a significant difference in their percentage between the groups (Fig. 2C). The differences were statistically significant $(\mathrm{P}<0.05)$ (Fig. 2D). 

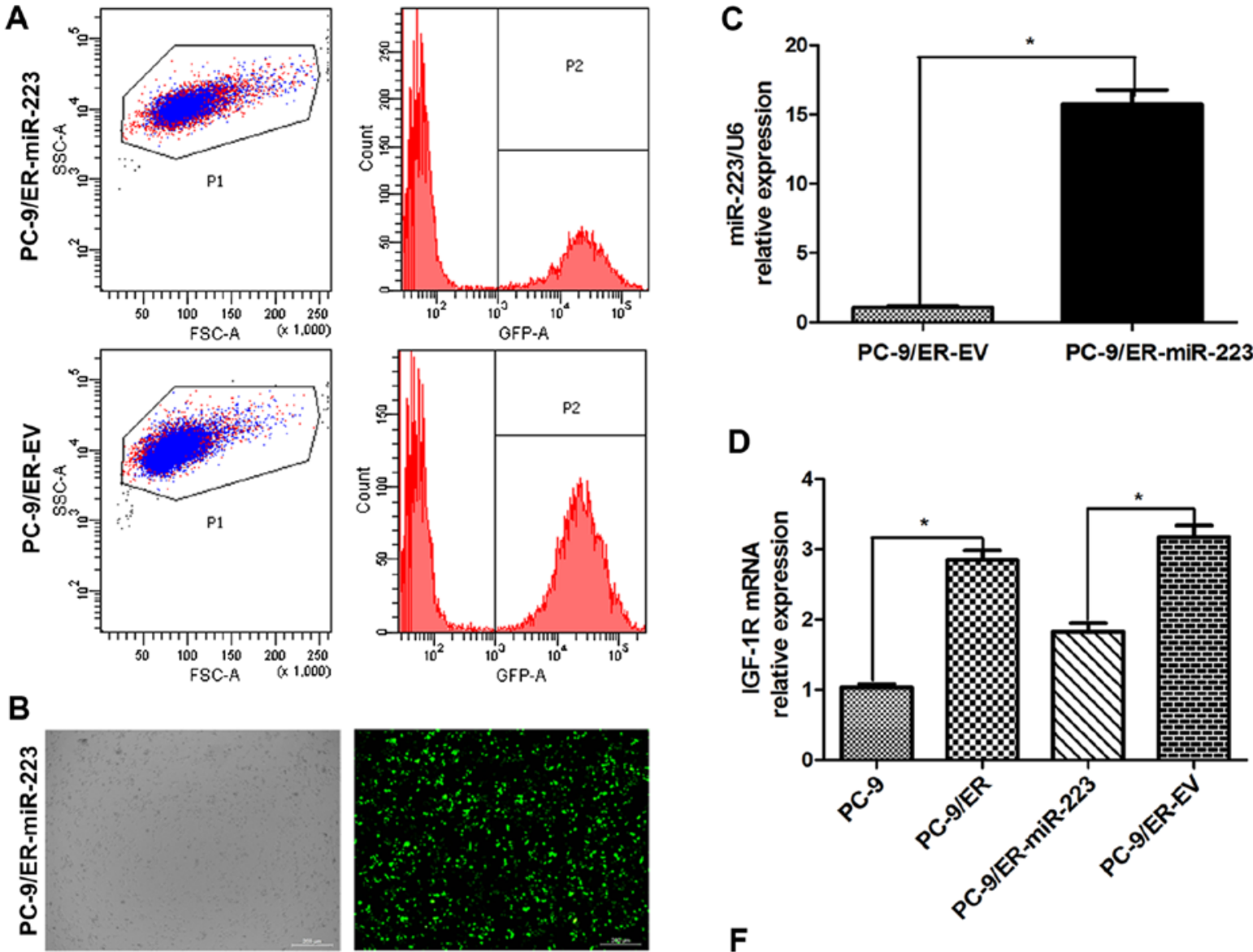

$\mathbf{F}$
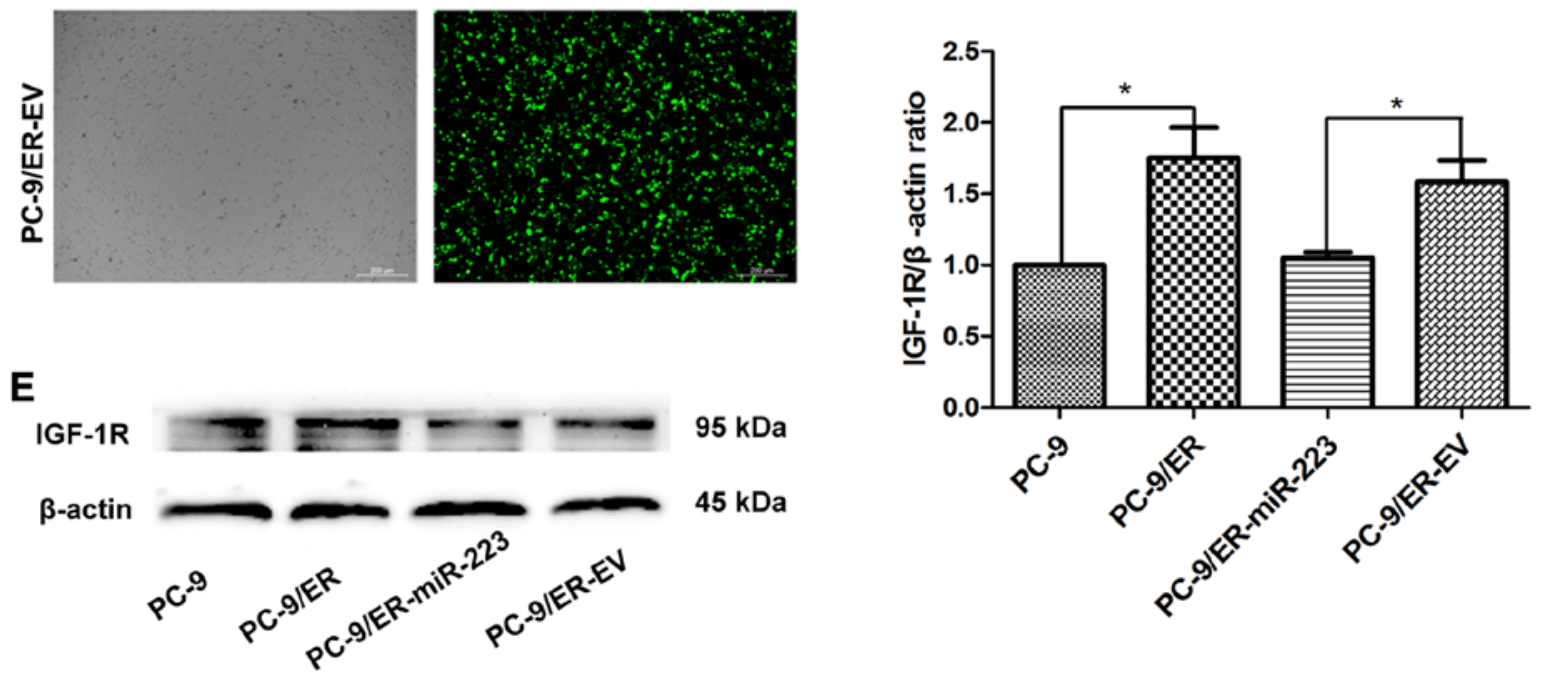

Figure 1. Lentivirus-mediated miR-223 overexpression and insulin-like growth factor-1 receptor (IGF-1R) expression in the PC-9 cell line. (A) The cells were sorted by fluorescence-activated cell sorting. (B) The infection efficiencies of PC-9/ER-miR-223 and PC-9/ER-EV cells were observed by fluorescence microscopy, based on the green fluorescent protein fluorescent signal after $72 \mathrm{~h}$. (C) miR-223 detection in the PC-9/ER cells by RT-qPCR. (D) The mRNA expression of IGF-1R was analyzed by RT-qPCR. (E) Western blot analysis of IGF-1R. (F) Histogram of panel (E) ( $\mathrm{n}=3$ experiments; $\left.{ }^{*} \mathrm{P}<0.05\right)$.

miR-223 inhibits IGF-1R/Akt/S6 signaling, and this effect is reversed by the exogenous expression of IGF-1. IGF-1R can be activated by the ligand IGF-1. The concentration and exposure time to IGF-1 were determined as follows: $60 \mathrm{~min}$ was the most appropriate duration to induce the re-expression of p-IGF-1R in the PC-9 cell line. The most appropriate concentration was $100 \mathrm{ng} / \mathrm{ml}$ (data not shown). We thus performed the following experiments under this condition. The levels of p-IGF-1R were significantly increased following stimulation with IGF-1 (Fig. 3A and B). As the mRNA and protein expression levels of IGF-1R were suppressed by miR-223, we wished to determine whether the IGF-1R-mediated downstream signaling pathway is also regulated by miR-223. The levels of total Akt (t-Akt) were unaffected; however, the levels of its active form (p-Akt) in the miR-223 overexpression group were reduced by approximately $65 \%$ compared with 

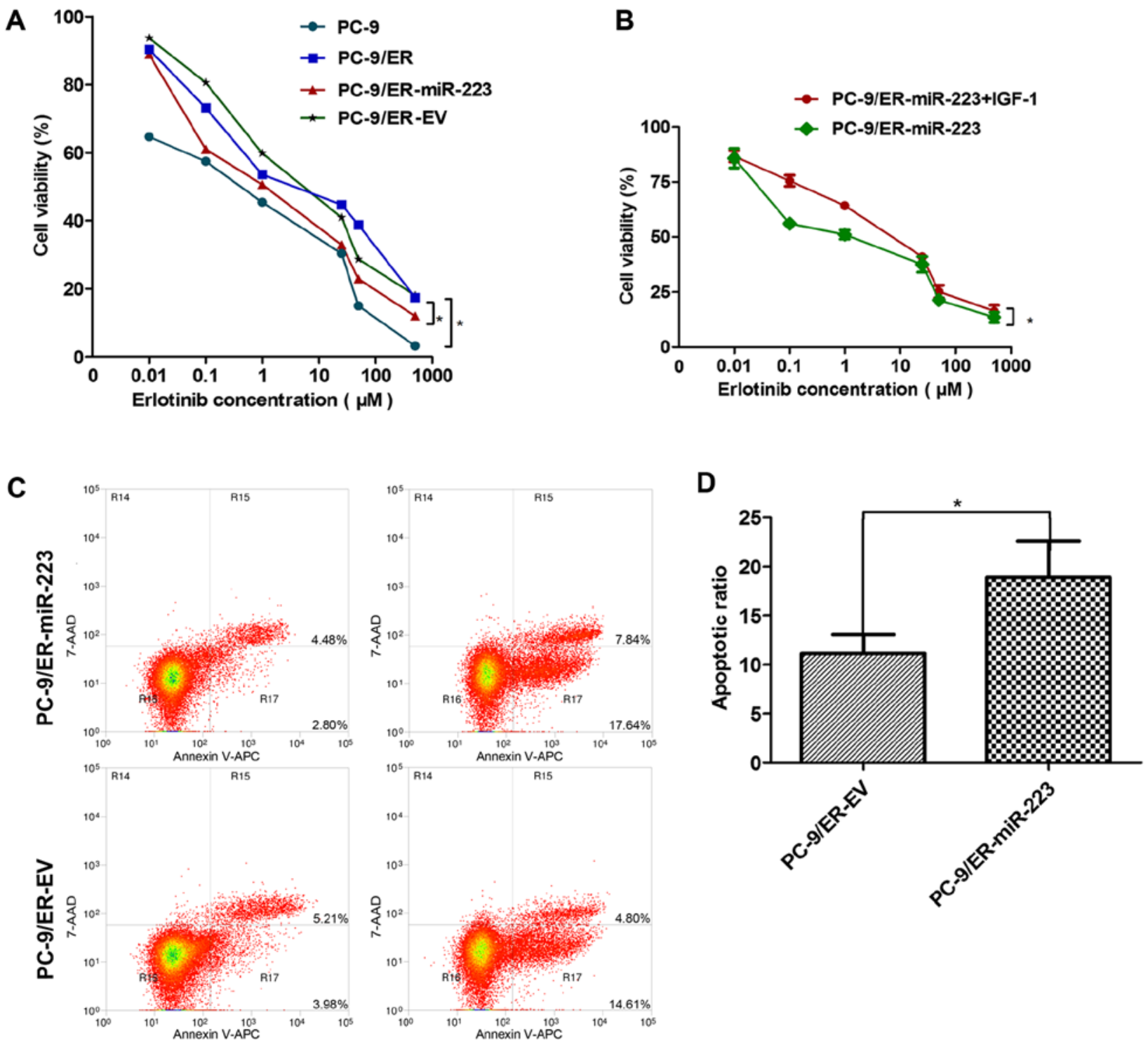

Figure 2. Overexpression of miR-223 enhances cell sensitivity to erlotinib by inducing apoptosis. (A) The sensitivity of the PC-9, PC-9/ER, miR-223 and EV-infected PC-9/ER cells to erlotinib was determined by cell counting kit-8 (CCK-8) assay. IC $_{50}$ of groups PC-9, PC-9/ER, PC-9/ER-miR-223 and PC-9/ER-EV: 0.25, 5.16,1.19 and 5.29. (B) The sensitivity to erlotinib of the PC-9/ER-miR-223 cells was assessed by CCK-8 assay in the presence or absence of insulin-like growth factor-1 (IGF-1) $(100 \mathrm{ng} / \mathrm{ml})$. (C and D) miR-223 enhanced cell apoptosis compared with the EV group following erlotinib treatment for $48 \mathrm{~h}$ as determined by flow cytometry. The apoptotic ratios were $18.92 \pm 2.123$ vs. $11.13 \pm 1.127 \%$. The data are expressed as the means \pm SD $\left(n=3\right.$ experiments; $\left.{ }^{~} \mathrm{P}<0.05\right)$.

those of the EV group (Fig. 3E). Furthermore, the levels of S6 and its active form, p-S6, were also detected. The levels of $\mathrm{p}-\mathrm{S} 6$ in the miR-223 group were markedly reduced by $45 \%$ compared with the EV group; however, the levels of total S6 were unaffected (Fig. 3F). The suppression of the Akt/S6 signaling pathway was consistently abolished by the exogenous expression of IGF-1. Therefore, miR-223 inhibited the IGF-1R/AKT/S6 signaling pathway by targeting IGF1R, and this inhibitory effect was reversed by IGF-1 in vitro (Fig. 3C).

miR-223 enhances the sensitivity to erlotinib and inhibits tumor growth in nude mice. To further confirm the above findings, an in vivo model was established by the subcutaneous injection of $2 \times 10^{6}$ PC-9/ER-miR-223- or PC-9/ER-EV-infected cells into the mouse skins under the left lower quadrants. Tumor sizes in the 6 groups were measured every 3 days. The tumor mass became palpable 6-9 days following inoculation in all groups. All the nude mice began to receive erlotinib treatment from the 14th day. Following the intragastric administration of erlotinib for 2 weeks, all mice were sacrificed and the tumor mass was measured (Fig. 4A). Tumor growth curves were plotted using GraphPad Prism 5 (Fig. 4B). The tumor volume of the PC-9/ER-injected mice was approximately 5 -fold larger than that of the PC-9-injected mice. The inhibition of tumor growth was observed in the PC-9/ER-miR-223 tumor-bearing nude mice. The tumor mass in the PC-9/ER-miR-223 group was $60 \%$ of the control $(\mathrm{P}<0.05)$. The PC-9/ER-miR-223 group with IGF-1 intervention exhibited a significant difference in tumor volume when compared with the control. The upregulation of miR-223 enhanced sensitivity to erlotinib and this was reversed by the exogenous expression of IGF-1. In survival analysis, 30 mice were used. Survival curves were produced using 

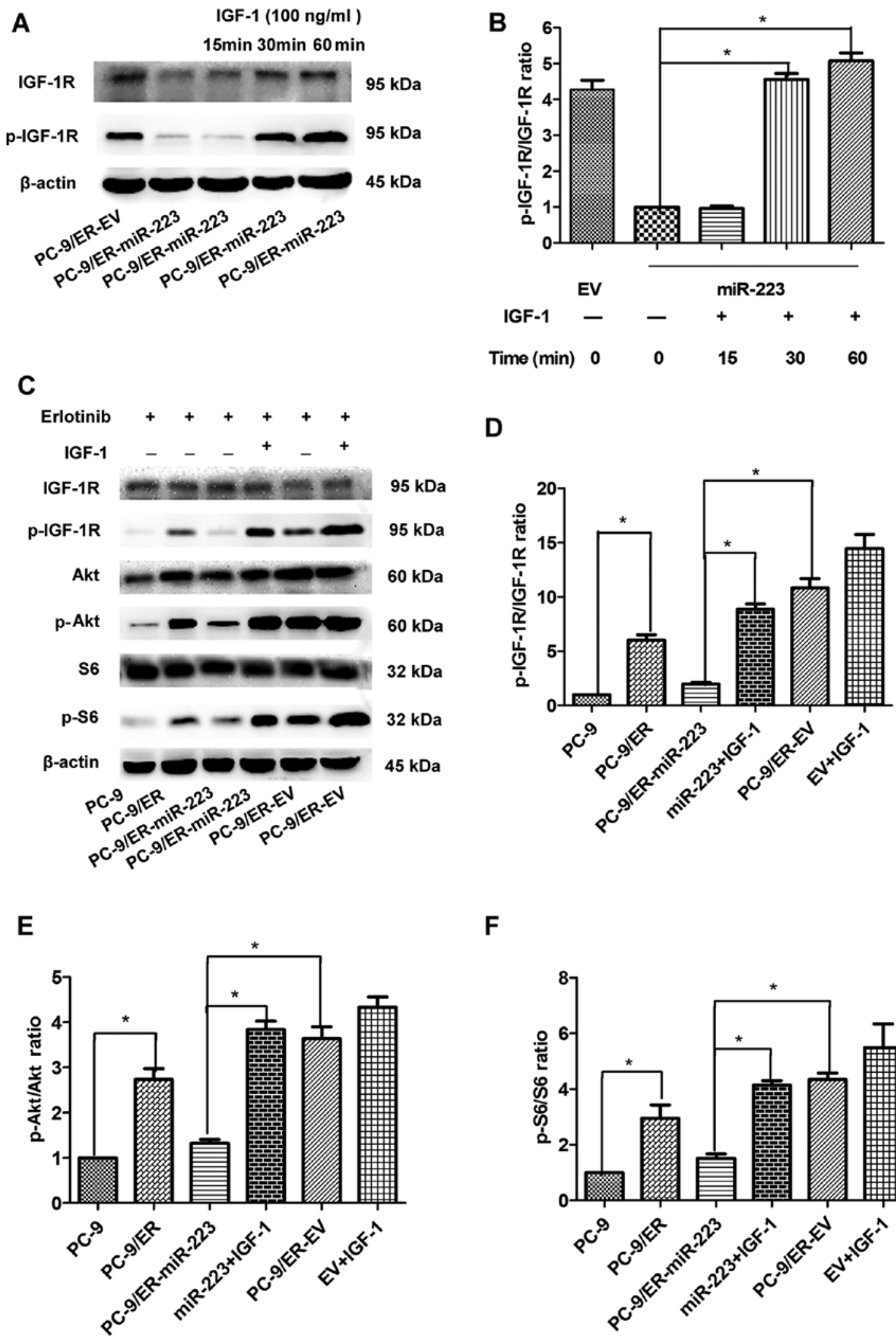

$\mathbf{F}$

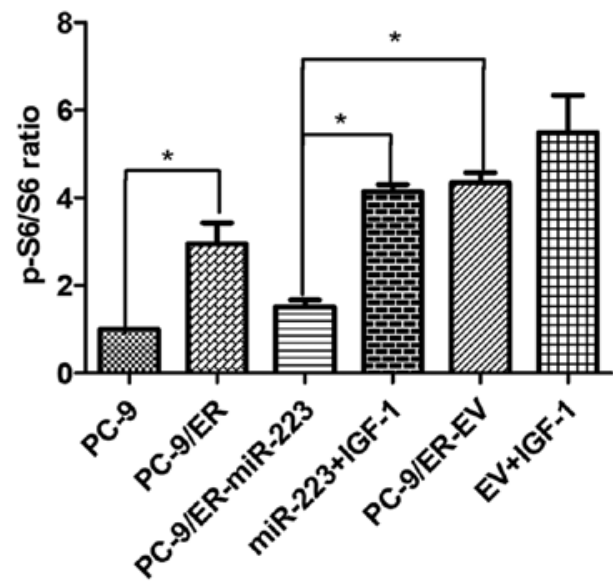

Figure 3. miR-223 inhibits insulin-like growth factor-1 receptor (IGF-1R)/Akt/S6 signaling by targeting IGF-1R and these inhibitory effects are reversed by exogenous IGF-1. (A and B) The protein levels of IGF-1R and p-IGF-1R in the miR-223- or EV-infected cells were detected at 0, 15, 30 and 60 min following stimulation with IGF-1. Statistical analysis of p-IGF-1R protein bands was performed following quantification using imaging analysis software. (C) Western blotting was performed to detect the expression levels of IGF-1R, p-IGF-1R, AKT, p-AKT, S6 and p-S6 in the PC-9 cell line following stimulation with IGF-1 for the indicated groups. Statistical analysis of the (D) p-IGF-1R, (E) p-AKT and (F) p-S6 protein bands was performed following quantification using imaging analysis software. The data are expressed as the means $\pm \mathrm{SD}\left(\mathrm{n}=3\right.$ experiments; $\left.{ }^{*} \mathrm{P}<0.05\right)$.

GraphPad Prism 5. A high expression of IGF-1R correlated with a poor survival rate $(\mathrm{P}<0.05)$. However, there was no difference between the exogenous IGF-1 group and the PC-9/ER-EV group in survival (Fig. 4C). 
A

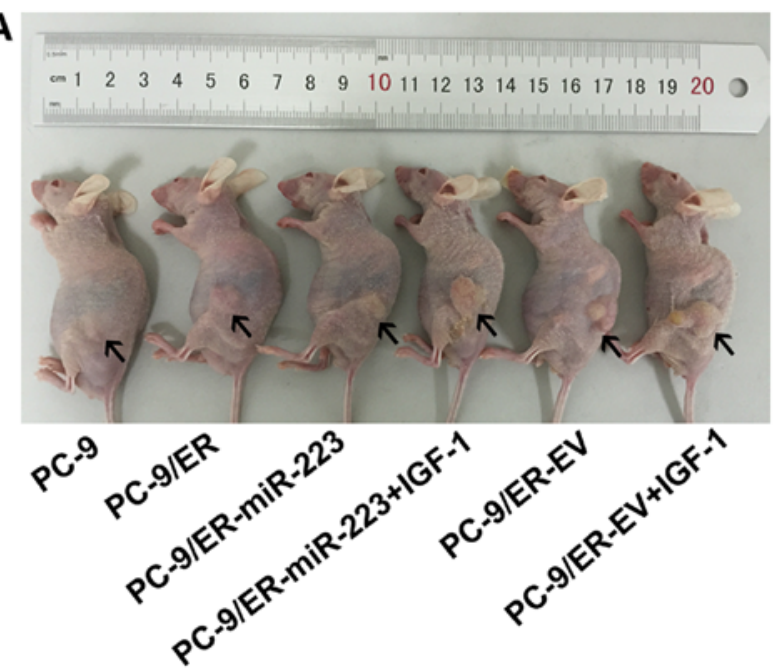

B

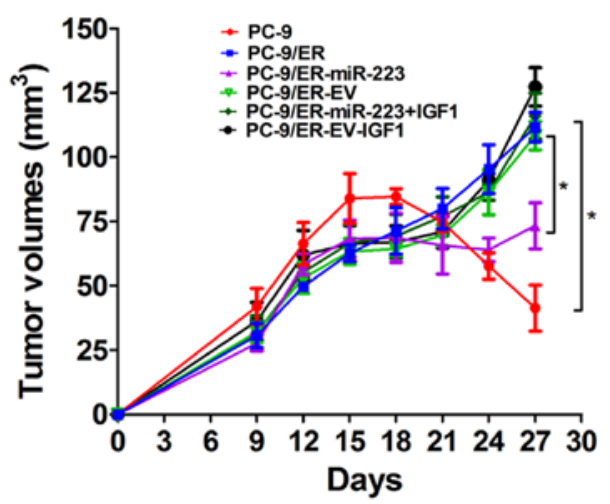

C

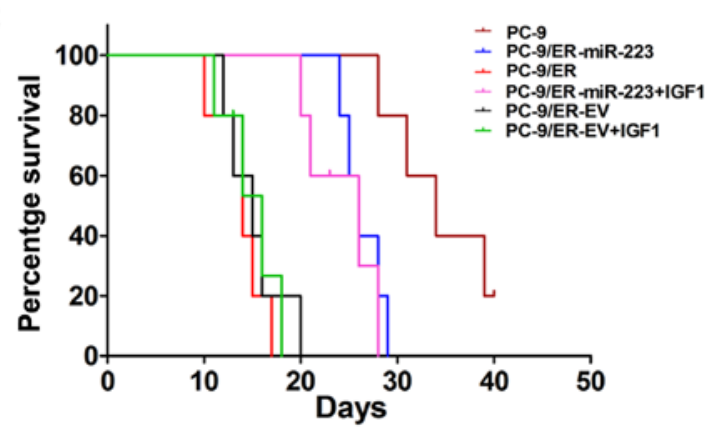

Figure 4. miR-223 improves the tumor response to erlotinib in tumor xenografts. (A) Representative nude mice bearing xenografts in 6 groups. All the mice received erlotinib via intragastric administration for 14 days. (B) Growth curves of tumors in 6 groups $\left(\mathrm{n}=3\right.$ mice per group; $\left.{ }^{*} \mathrm{P}<0.05\right)$. (C) Survival curves of nude mice in 6 groups $(n=30)$. Continuous intragastric administration until morbidity or death ( $\mathrm{n}=5$ mice per group; $\mathrm{P}<0.05)$.

\section{Discussion}

miRNAs have been reported to play important roles in tumor initiation and progression (24). The up- or downregulation of miRNAs are predominantly investigated in cancer biology. miR-223 was initially identified and subsequently characterized in the hematopoietic system (25). Previous studies have revealed that miR-223 is expressed at a low level in nasopharyngeal carcinoma cells (26), undifferentiated human embryonic stem cells (hESCs) (27) and HeLa cells (15). Furthermore, miR-223 often suppresses cell growth, colony formation and proliferation (15). Numerous studies have confirmed that miR-223 plays an important role in lung cancer $(28,29)$. However, its role in resistance to TKIs in targeted molecular therapy in NSCLC remains unknown. The mechanisms by which miR-223 regulates resistance to erlotinib also remain to be elucidated. In the present study, we established a lentivirus-based miR-223 overexpression system. Several important methods were used to examine the association between miR-223 and IGF-1R, and the downstream signaling pathway of miR-223.

In our previous study, we found that IGF-1R is a target of miR-223 (16). A significant difference was observed in the response to erlotinib between the PC-9 and PC-9/ER cells, or the miR-223- and EV-infected groups. We found that miR-223 was a positive regulator or promoter of tumor cell apoptosis, which enhanced the sensitivity of the cells to erloninib by targeting the IGF-1R/Akt/S6 signaling pathway in vitro. In vivo, miR-223 overexpression enhanced the sensitivity to erlotinib, as the tumor volume of mice injected with miR-223-infected cells was lower than that of the mice injected with PC-9/ER cells. In addition, the nude mice injected with miR-223-infected cells survived longer than the control mice.

Despite the promising effects of TKIs, the acquired resistance must be overcome. The persistent activation of downstream signaling pathways, particularly PI3K/Akt, is sufficient to confer resistance to cells against EGFR-TKIs by bypassing EGFR blocking (30). To elucidate the underlying mechanisms involved in the miR-223-induced apoptosis of PC-9/ER cells, we used different prediction algorithms to predict the target genes of miR-223. IGF-1R was predicted to be one such target. IGF-1R has been well characterized in resistance to TKIs in NSCLC. The regulation of IGF-1R signaling is receiving increasing attention in the development of targeted therapeutics to improve human health. IGF-1R activates the Ras/Erk- and PI3K/Akt-associated signal transduction pathways (31). The present study revealed that IGF-1R expression was upregulated in the cells with acquired resistance to erlotinib at the mRNA and protein level. By contrast, the mRNA expression level of IGF-1R in H1975 cells with T790M mutation was lower than that in PC-9/ER cells (data not shown). These results suggested that IGF-1R may play an important role in the acquired resistance to TKIs than in intrinsic resistance. In addition, it was demonstrated that the binding of IGF-1 to IGF-1R stimulated tumor growth by activating anti-apoptotic signaling pathways.

Techniques to inhibit IGF-1R have been investigated as promising therapeutic strategies against resistance to TKIs in NSCLC. To date, IGF-1R antibodies, including dalotuzumab (MK-0646), have been reported to be safe and able to reduce IGF-1R signaling in phase I and II clinical trials. The clinical activity of IGF-1R inhibitors has been demonstrated with sustained responses in a number of patients. However, in adult patients with tumors, including NSCLC, breast cancer and pancreatic cancer, have failed to reveal the clinical benefit of IGF-1R inhibitors in the overall patient population $(32,33)$. In those studies, it was assumed that the level of IGF-1R activation in patients correlated with the efficacy of dalotuzumab. Therefore, we hypothesized that a highly activate IGF-1R is essential for developing its functions. However, in the present study, IGF-1R expression was much higher in the PC-9/ER cells than in the PC-9 cells, indicating that TKI-resistant patients are possibly suitable candidates for MK-0646. Patients with 
TKI-resistant NSCLC may benefit from dual drug therapy with EGFR-TKIs and dalotuzumab. High levels of circulating IGF-1 are known to correlate with increased risks of breast, prostate and colon cancers (34). IGF-1 is an ubiquitously produced protein hormone, which interacts with IGF-1R to regulate cell growth, differentiation and survival (35). Ligand binding fails to stimulate the kinase activity of phosphorylated IGF-1R; however, it does stimulate IGF-1R autophosphorylation (36). Removing or inhibiting the secretion of IGF-1 in plasma may reverse cell tolerance to TKIs.

In conclusion, the present study hypothesized that combined EGFR/IGF-1R inhibition may improve the efficacy of targeted molecular therapy in erlotinib-resistant NSCLC. IGF-1R is a valid target for selected tumor types, including erlotinib-resistant lung cancer with a low expression of miR-223. By contrast, miR-223, an evolutionarily conserved miRNA, represents a potential biomarker for erlotinib-resistant NSCLC. Thus, the overexpression of miR-223 in TKI-resistant NSCLC may prove to be beneficial. The present study described the role of the IGF/IGF-1R system, and proposed additional novel strategies for targeting this system. Strategies to target this system have also been proposed previously (37). These results indicate the great potential of miRNAs in the development of therapeutics. Despite the limitations in delivery and toxicity, this study may provide a vital miRNA target for combating resistance to TKIs in the treatment of NSCLC.

\section{Acknowledgements}

The authors would like to express their gratitude to Mr. Dian-Gang Chen (Cancer Institute of PLA, Xinqiao Hospital, Third Military Medical University, China) for kindly providing the $\mathrm{H} 1975$ cell line and instructing the experimental skills. We should express our gratitude to the premium language editing service of Spandidos Publications. The present study was supported by the National Natural Science Foundation of China (grant no. 81172070), the Chongqing Science and Technology Key Project Fund (grant no. 2011AB5032), the Military Clinical Key Projects of New and High Technology (grant no. 2010gxjs070) and the Natural Science Foundation of Chongqing (grant no. cstc2012jjA10096).

\section{References}

1. Jemal A, Siegel R, Xu J and Ward E: Cancer statistics, 2010. CA Cancer J Clin 60: 277-300, 2010.

2. Allemani C, Weir HK, Carreira H, Harewood R, Spika D, Wang XS, Bannon F, Ahn JV, Johnson CJ, Bonaventure A, et al; CONCORD Working Group: Global surveillance of cancer survival 1995-2009: Analysis of individual data for 25,676,887 patients from 279 population-based registries in 67 countries (CONCORD-2). Lancet 385: 977-1010, 2015.

3. Larsen JE and Minna JD: Molecular biology of lung cancer: Clinical implications. Clin Chest Med 32: 703-740, 2011.

4. Engelman JA, Zejnullahu K, Mitsudomi T, Song Y, Hyland C, Park JO, Lindeman N, Gale CM, Zhao X, Christensen J, et al: MET amplification leads to gefitinib resistance in lung cancer by activating ERBB3 signaling. Science 316: 1039-1043, 2007.

5. Morgillo F, Kim WY, Kim ES, Ciardiello F, Hong WK and Lee HY: Implication of the insulin-like growth factor-IR pathway in the resistance of non-small cell lung cancer cells to treatment with gefitinib. Clin Cancer Res 13: 2795-2803, 2007.

6. Del Vescovo V and Denti MA: microRNA and lung cancer. Adv Exp Med Biol 889: 153-177, 2015.

7. Lagos-Quintana M, Rauhut R, Lendeckel W and Tuschl T: Identification of novel genes coding for small expressed RNAs. Science 294: 853-858, 2001.
8. Del Vescovo V, Grasso M, Barbareschi M and Denti MA: MicroRNAs as lung cancer biomarkers. World J Clin Oncol 5: 604-620, 2014.

9. Lee CT, Risom T and Strauss WM: MicroRNAs in mammalian development. Birth Defects Res C Embryo Today 78: 129-139, 2006.

10. Bueno MJ, Pérez de Castro I and Malumbres M: Control of cell proliferation pathways by microRNAs. Cell Cycle 7: 3143-3148, 2008.

11. Jovanovic $M$ and Hengartner MO: miRNAs and apoptosis: RNAs to die for. Oncogene 25: 6176-6187, 2006.

12. Mestdagh P, Hartmann N, Baeriswyl L, Andreasen D, Bernard N, Chen C, Cheo D, D'Andrade P, DeMayo M, Dennis L, et al: Evaluation of quantitative miRNA expression platforms in the microRNA quality control (miRQC) study. Nat Methods 11: 809-815, 2014.

13. Garofalo M, Quintavalle C, Di Leva G, Zanca C, Romano G, Taccioli C, Liu CG, Croce CM and Condorelli G: MicroRNA signatures of TRAIL resistance in human non-small cell lung cancer. Oncogene 27: 3845-3855, 2008.

14. Wong QW, Lung RW, Law PT, Lai PB, Chan KY, To KF and Wong N: MicroRNA-223 is commonly repressed in hepatocellular carcinoma and potentiates expression of Stathmin1. Gastroenterology 135: 257-269, 2008.

15. Jia CY, Li HH, Zhu XC, Dong YW, Fu D, Zhao QL, Wu W and Wu XZ: MiR-223 suppresses cell proliferation by targeting IGF-1R. PLoS One 6: e27008, 2011.

16. Nian W, Ao X, Wu Y, Huang Y, Shao J, Wang Y, Chen Z, Chen F and Wang D: miR-223 functions as a potent tumor suppressor of the Lewis lung carcinoma cell line by targeting insulin-like growth factor-1 receptor and cyclin-dependent kinase 2. Oncol Lett 6: 359-366, 2013.

17. Peled N, Wynes MW, Ikeda N, Ohira T, Yoshida K, Qian J, Ilouze M, Brenner R, Kato Y, Mascaux C and Hirsch FR: Insulin-like growth factor-1 receptor (IGF-1R) as a biomarker for resistance to the tyrosine kinase inhibitor gefitinib in non-small cell lung cancer. Cell Oncol (Dordr) 36: 277-288, 2013.

18. Baserga R, Peruzzi F and Reiss K: The IGF-1 receptor in cancer biology. Int J Cancer 107: 873-877, 2003.

19. Pollak MN, Schernhammer ES and Hankinson SE: Insulin-like growth factors and neoplasia. Nat Rev Cancer 4: 505-518, 2004.

20. Wang Q, Zhao DY, Xu H, Zhou H, Yang QY, Liu F and Zhou GP: Downregulation of microRNA-223 promotes degranulation via the PI3K/Akt pathway by targeting IGF-1R in mast cells. PLoS One 10: e0123575, 2015.

21. Yuan Y, Shen Y, Xue L and Fan H: miR-140 suppresses tumor growth and metastasis of non-small cell lung cancer by targeting insulin-like growth factor 1 receptor. PLoS One 8: e73604, 2013.

22. Agulló-Ortuño MT, Díaz-García CV, Agudo-López A, Pérez C, Cortijo A, Paz-Ares L, López-Ríos F, Pozo F, de Castro J and López Martín JA: Relevance of insulin-like growth factor 1 receptor gene expression as a prognostic factor in non-small-cell lung cancer. J Cancer Res Clin Oncol 141: 43-53, 2015.

23. Scherr M, Venturini L and Eder M: Lentiviral vector-mediated expression of pre-miRNAs and antagomiRs. Methods Mol Biol 614: 175-185, 2010.

24. Ryan BM, Robles AI and Harris CC: Genetic variation in microRNA networks: The implications for cancer research. Nat Rev Cancer 10: 389-402, 2010.

25. Chen CZ, Li L, Lodish HF and Bartel DP: MicroRNAs modulate hematopoietic lineage differentiation. Science 303: 83-86, 2004.

26. Yang W, Lan X, Li D, Li T and Lu S: MiR-223 targeting MAFB suppresses proliferation and migration of nasopharyngeal carcinoma cells. BMC Cancer 15: 461, 2015.

27. Yu YH, Zhang L, Wu DS, Zhang Z, Huang FF, Zhang J, Chen XP, Liang DS, Zeng $\mathrm{H}$ and Chen FP: MiR-223 regulates human embryonic stem cell differentiation by targeting the IGF-1R/Akt signaling pathway. PLoS One 8: e78769, 2013.

28. Sanfiorenzo C, Ilie MI, Belaid A, Barlési F, Mouroux J, Marquette $\mathrm{CH}$, Brest $\mathrm{P}$ and Hofman P: Two panels of plasma microRNAs as non-invasive biomarkers for prediction of recurrence in resectable NSCLC. PLoS One 8: e54596, 2013.

29. Heegaard NH, Schetter AJ, Welsh JA, Yoneda M, Bowman ED and Harris CC: Circulating micro-RNA expression profiles in early stage nonsmall cell lung cancer. Int J Cancer 130: $1378-1386,2012$. 
30. Yamasaki F, Johansen MJ, Zhang D, Krishnamurthy S, Felix E, Bartholomeusz C, Aguilar RJ, Kurisu K, Mills GB, Hortobagyi GN and Ueno NT: Acquired resistance to erlotinib in A-431 epidermoid cancer cells requires downregulation of MMAC1/PTEN and upregulation of phosphorylated Akt. Cancer Res 67: 5779-5788, 2007.

31. Bohula EA, Playford MP and Macaulay VM: Targeting the type 1 insulin-like growth factor receptor as anti-cancer treatment. Anticancer Drugs 14: 669-682, 2003.

32. Scartozzi M, Bianconi M, Maccaroni E, Giampieri R, Berardi R and Cascinu S: Dalotuzumab, a recombinant humanized $\mathrm{mAb}$ targeted against IGFR1 for the treatment of cancer. Curr Opin Mol Ther 12: 361-371, 2010.

33. Reidy-Lagunes DL, Vakiani E, Segal MF, Hollywood EM, Tang LH, Solit DB, Pietanza MC, Capanu M and Saltz LB: A phase 2 study of the insulin-like growth factor-1 receptor inhibitor MK-0646 in patients with metastatic, well-differentiated neuroendocrine tumors. Cancer 118: 4795-4800, 2012.
34. Pollak M: Insulin and insulin-like growth factor signalling in neoplasia. Nat Rev Cancer 8: 915-928, 2008.

35. Jameson MJ, Beckler AD, Taniguchi LE, Allak A, Vanwagner LB, Lee NG, Thomsen WC, Hubbard MA and Thomas CY: Activation of the insulin-like growth factor-1 receptor induces resistance to epidermal growth factor receptor antagonism in head and neck squamous carcinoma cells. Mol Cancer Ther 10: 2124-2134, 2011.

36. Kavran JM, McCabe JM, Byrne PO, Connacher MK, Wang Z Ramek A, Sarabipour S, Shan Y, Shaw DE, Hristova K, et al: How IGF-1 activates its receptor. Elife 3: 3, 2014.

37. Brahmkhatri VP, Prasanna C and Atreya HS: Insulin-like growth factor system in cancer: Novel targeted therapies. BioMed Res Int 2015: 538019, 2015. 